\title{
Avaliação nacional dos centros de referência odontológica para atendimento de pacientes com necessidades especiais
}

\author{
National evaluation of reference dental centers for the care of patients with special \\ needs
}

\section{Evaluación nacional de los centros de referencia odontológica para atención de pacientes con necesidades especiales}

\author{
Recebido: 08/07/2017 \\ Aprovado: 05/12/2017 \\ Publicado: 05/04/2018
}

\author{
Renato Carvalho Morais Junior ${ }^{1}$ \\ Laís Guedes Alcoforado de Carvalho \\ Isabella Lima Arrais Ribeiro ${ }^{3}$ \\ Ricardo Dias de Castro ${ }^{4}$
}

O presente estudo teve como objetivo avaliar nas capitais brasileiras a quantidade de Centros de Especialidades Odontológicas (CEOs) habilitados como "Rede de Cuidados à Pessoa com Deficiência" (CEO-RCPD), e se esse número é compatível para atendimento da demanda existente de pacientes com necessidades especiais (PNEs) em cada uma das capitais. Trata-se de um estudo transversal, quantitativo, de caráter avaliativo normativo da relação entre o número de CEOs e o número de PNEs das capitais brasileiras no ano de 2017. De acordo como Ministério da Saúde, o Brasil possui 133 CEOs atuando nas capitais brasileiras. Dentre estes, apenas 79 estão habilitados a atender a demanda dos pacientes com necessidades especiais. A região Centro-Oeste foi a que apresentou a maior oferta de atendimento a PNEs nos CEOs $(87,5 \%)$, e a região Sul, a menor $(11,1 \%)$. Os resultados sugerem a necessidade de aumentar o número de CEOs que atendam a PNEs, de forma a assistir integral e universalmente a população brasileira em suas necessidades de saúde bucal, bem como reduzir o tempo de espera dos usuários e a necessidade de deslocamento para outros municípios ou Estados.

Descritores: Saúde bucal; Sistemas de informação em saúde; Recursos humanos em odontologia.

This study aimed to evaluate the number of Odontological Specialty Centers (CEOs) qualified as part of the "Network of Care for People with Disabilities" (RCPD-CEO), and whether this number is adequate to cater for the demand of patients with special needs (PSNs) in each capital. This is a cross-sectional, quantitative, and normativeevaluative study, of the relation between the number of CEOs and the number of PSNs in the Brazilian capitals in 2017. According to the Ministry of Health, there are 133 CEOs in the Brazilian capitals. Among these, only 79 are qualified to care for the patients with special needs. The Brazilian Midwest had the most centers offering attention to PENs (87.5\%), and the south, the least (11.1\%). The results suggest the need to increase the number of CE0s that attend PENs, as to integrally and universally assist the Brazilian population in their oral health needs, as well as to diminish the waiting time of users and their need to go to other cities or states in order to be treated.

Descriptors: Oral health; Health information systems; Dental staff.

El presente estudio tuvo como objetivo evaluar en las capitales brasileras la cantidad de Centros de Especialidades Odontológicas (CEOs) habilitados como "Red de Cuidados a la Persona con Deficiencia" (CEO-RCPD), y si ese número es compatible para la atención de la demanda existente de pacientes con necesidades especiales (PNEs) en cada una de las capitales. Se trata de un estudio transversal, cuantitativo, de carácter evaluativo normativo de la relación entre el número de CEOs y el número de PNEs de las capitales brasileras en el año de 2017. De acuerdo con el Ministerio de la Salud, Brasil posee 133 CEOs actuando en las capitales brasileras. Entre estos, solo 79 están habilitados para atender la demanda de los pacientes con necesidades especiales. La región Centro-Oeste fue la que presentó la mayor oferta de atención a PNEs en los CEOs (87,5\%), y la región Sur, la menor (11,1\%). Los resultados sugieren la necesidad de aumentar el número de CEOs que atiendan a los PNEs, de forma a asistir integral e universalmente a la población brasilera en sus necesidades de salud bucal, así como reducir el tiempo de espera de los usuarios y la necesidad de traslado para otros municipios o Estados.

Descriptores: Salud bucal; Sistemas de información en salud; Personal de odontología.

1. Cirurgião Dentista. Especialista em Pacientes Portadores de Necessidades Especiais. Dentista do Centro de Especialidades Odontológicas (CE0). João Pessoa-PB, Brasil. ORCID: 0000-0002-3572-6843 E-mail: renatocarvalho@outlook.com

2. Cirurgiã Dentista. Mestre em Ciências Odontológicas. João Pessoa-PB, Brasil. ORCID: 0000-0003-2615-2582 E-mail:laisgac@gmail.com

3 Cirurgião Dentista. Especialista em Endodontia. Mestre em Diagnóstico Bucal. Doutora em Modelos de Decisão e Saúde. Pós-Doutora em Epidemiologia, Universidade Federal da Paraíba (UFPB), João Pessoa, PB, Brasil. ORCID: 0000-0001-6538-6811 E-mail: isabella_arrais@yahoo.com

4. Cirurgião Dentista. Mestre em Odontologia Preventiva e Social. Doutor em Farmacologia. Pós-Doutor em Odontologia. Professor Adjunto do Departamento de Clínica e Odontologia Social da UFPB, João Pessoa, PB, Brasil. ORCID: 0000-0001-7986-7376 E-mail: ricardodiasdecastro@yahoo.com.br 


\section{INTRODUÇÃO}

D e acordo com as diretrizes, princípios e funcionalidades do Sistema Único de Saúde (SUS), em 2003, a Política Nacional de Saúde Bucal (PNSB) foi criada, tendo como principal objetivo a reorganização da prática e a qualificação das ações e serviços oferecidos, reunindo uma série de ações em saúde bucal voltadas aos cidadãos de todas as idades, com ampliação do acesso ao tratamento odontológico gratuito aos brasileiros ${ }^{1}$.

Apesar da melhoria do atendimento odontológico ano após ano, notou-se que a demanda de serviços e atendimento especializado, que não se conseguia executar na atenção básica, foi sendo cada vez mais exigida:

"Com a expansão do conceito de atenção básica, e o consequente aumento da oferta de diversidade de procedimentos, fazem-se necessários, também, investimentos que propiciem aumentar o acesso aos níveis secundário e terciário de atenção. Para fazer frente ao desafio de ampliar e qualificar a oferta de serviços odontológicos especializados foi criado o Centro de Especialidades Odontológicas - CEO" Diretrizes da Política Nacional de Saúde Bucal'.

No ano de 2004, foram criados os Centros de Especialidades Odontológicas CEO, que são classificados como Clínica Especializada ou Ambulatório de Especialidade, atualmente oferecendo à população proveniente da Atenção Básica, as seguintes especialidades: Periodontia; Diagnóstico Bucal com ênfase no diagnóstico precoce e detecção do câncer bucal; Cirurgia Oral Menor; Endodontia; e Atendimento a Pacientes com Necessidades Especiais ${ }^{2}$.

0 atendimento nos CEOs é realizado de forma programada, após o paciente ser referenciado pelo profissional da atenção básica (ESF), que avalia a real necessidade do encaminhamento apenas em casos mais complexos ${ }^{3}$.

Os Centros de Especialidades odontológicas podem ser classificados em três tipos: CEO tipo I (três cadeiras odontológicas), CEO tipo II (Quatro a seis cadeiras odontológicas) e CEO tipo III (possuem, no mínimo, sete cadeiras). Em relação à quantidade de funcionários em cada CEO, há uma grande variabilidade, de acordo com a demanda e necessidades locais de cada Município ou Unidade, mas todos devem funcionar 40 horas semanais 4 .

A implantação dos CEOs constitui uma estratégia relevante, visando a integridade da atenção na área odontológica. Não apenas a implantação dessas unidades da média complexidade (ou atenção secundária) se faz importante para a melhoria gradativa da saúde bucal dos brasileiros, mas também o incentivo por parte do governo Federal, incluindo incentivos orçamentários, é de fundamental importância para o avanço, bem como para a melhoria constante de instalações e equipamentos utilizados nos CEOs $^{5}$.

Para esse avanço, o Programa Nacional de Melhoria do Acesso e da Qualidade (PMAQ) foi criado em 2011, e vem sendo utilizado desde então nas Equipes de Saúde Bucal na Atenção primaria. Segundo o Ministério da Saúde, o PMAQ é um programa de abrangência nacional que possui a finalidade de promover a melhoria do acesso e da qualidade da atenção à saúde 6 .

Desde o ano de 2013, o PMAQ também foi ampliado para os Centros de Especialidades Odontológicas, sendo denominado de PMAQ-CEO. Com a adesão ao programa, as equipes passam a receber $20 \%$ do recurso total designado a cada equipe participante. Após a avaliação externa, as equipes poderão perder o incentivo, mantê-lo ou ampliar para $60 \%$ ou $100 \%$, de acordo com o desempenho. Com isso, um centro odontológico tipo I, que recebe hoje $\mathrm{R} \$ 8.250$, vai receber R\$9.900. Já os CEOs tipo II irão passar a receber $\mathrm{R} \$ 13.200$ e as unidades tipo III saíram de $R \$ 19.250$ para $R \$ 23.100^{6}$.

Ainda no ano de 2012, foi criada a Rede de Cuidados à Pessoa com Deficiência através da portaria GM/MS no. 793 de 24 de abril 7 , que tem como objetivo:

"Criação, ampliação e articulação de pontos de atenção à saúde para pessoas com deficiência temporária ou permanente; progressiva, regressiva, ou estável; intermitente ou contínua, no âmbito do Sistema Único de Saúde (SUS)".

Através dessa Rede de Cuidados à Pessoa com Deficiência (RCPD), a Especialidade de Atendimento a Pacientes 
com Necessidades Especiais - PNE, começa a fazer parte dos CEOs de todo território nacional, sendo inclusive criados incentivos para os CEOs que fizerem adesão junto com a RCPD, que segue a seguinte ordem até os dias de hoje: $\mathrm{R} \$ 1.650,00$ mensais para o CEO tipo I; $\mathrm{R} \$ 2.200,00$ mensais para o CEO tipo II e $\mathrm{R} \$$ 3.850,00 mensais para o CEO tipo III ${ }^{7}$.

Esse incentivo nacional pode ser solicitado por todos os CEOs do país. Para tanto, eles devem cumprir as seguintes exigências ${ }^{7}$ :

- Atendimento de no mínimo 40 horas semanais exclusivo para PNEs;

- Oferecer apoio matricial para as Equipes de Saúde Bucal da Atenção Básica, no tocante ao atendimento e encaminhamento de pessoas com deficiência que necessitam de atendimento odontológico especializado;

- Manter alimentação regular e consistente da informação de produção do CEO por meio do Sistema de Informação Ambulatorial do SUS (SIA/SUS), utilizando como instrumento de registro o Boletim de Produção Ambulatorial Individualizado (BPA-I) para todos os procedimentos realizados em pessoas com deficiência;

- Manter a produção mensal mínima exigida na Portaria no 1.464/GM/MS, de 24 de junho de 2011; Aplicar os recursos adicionais do Ministério da Saúde pela incorporação do CEO à Rede de Cuidados à Pessoa com Deficiência exclusivamente para esse fim;

- Garantir condições de acessibilidade e mobilidade nas instalações do CEO para pessoas com deficiência;

- Prover o(s) CEOs com profissionais de saúde bucal capacitados para o atendimento odontológico de pessoas com deficiência7.

Após solicitação do gestor municipal ou estadual, a proposta é enviada para a Coordenação Geral de Saúde Bucal/DAB/SAS do Ministério da Saúde (MS) e para a
Comissão Intergestores Bipartite (CIB). Em seguida o MS publicará uma portaria específica, mudando a habilitação do CEO para CEO-RCPD? ${ }^{7}$.

Conforme Freitas et al.9:

"A implantação dos CEOs é uma estratégia relevante com vistas à integralidade da atenção no âmbito odontológico como serviços de saúde que representam, devem ser avaliados, objetivando a melhoria contínua da qualidade do serviço ofertado".

Dessa forma, o objetivo deste estudo foi avaliar a quantidade de CEOs nas capitais brasileiras, quantos são habilitados como CEO-RCPD, e se esse número é adequado para o atendimento do público-alvo com necessidades especiais.

\section{MÉTODO}

Trata-se de um estudo transversal, quantitativo, de caráter avaliativo normativo da relação entre o número de CEOs e o número de PNEs das capitais brasileiras no ano de 2017.

De acordo com o Ministério da Saúde, em 2017 havia 133 CEOs atuando nas capitais brasileiras. Dentre estes, apenas 79 estavam habilitados para atender a demanda dos pacientes com necessidades especiais.

Para a escolha dos municípios pesquisados, foram adotados os seguintes critérios de inclusão: Todas as capitais dos estados brasileiros e do Distrito Federal; CEOs dos tipos I, II e III, e CEOs que estivessem listados no banco de dados do DAB Departamento da Atenção Básica no ano de 2017.

A amostra foi constituída por 27 cidades (capitais brasileiras), variando de 1 a 28 CEOs em cada uma dessas cidades, independente da existência ou não de habilitação para o atendimento a PNEs e agrupadas por região, de acordo com a Tabela 1.

Tabela 1. Regiões Pesquisadas com CEO de acordo com o DAB, Brasil, 2017.

\begin{tabular}{cccc}
\hline Região & no.. capitais & CEOs Gerais & CEOs com PNE \\
\hline Centro-Oeste & 04 & 24 & $21(87,5 \%)$ \\
Nordeste & 09 & 32 & $21(65,6 \%)$ \\
Norte & 07 & 21 & $08(38,0 \%)$ \\
Sudeste & 04 & 47 & $28(59,5 \%)$ \\
Sul & 03 & 09 & $01(11,1 \%)$ \\
\hline
\end{tabular}


Esses dados foram obtidos através de páginas eletrônicas oficiais do IBGE e do SUS, e tabulados em uma planilha do Microsoft Excel versão 2017, através da qual se fez a consolidação dos dados de acordo com a capital e a região brasileira, podendo-se analisa-la por capital ou formar um subgrupo das 5 regiões brasileiras. Após tabulação, a análise estatística foi feita no software IBM SPSS (20.0).

Das variáveis independentes foram consideradas: População da capital segundo estimativa do IBGE para 2016; Pessoas sem nenhum tipo de deficiência; Pessoas com pelo menos 1 tipo de deficiência; Porcentagem de pessoas com necessidades especiais; Quantidade de CEOs que fazem parte do programa Viver Sem Limites.

\section{RESULTADOS}

O Gráfico 1 mostra a relação entre a população que não possui nenhum tipo de deficiência e o número de pessoas com pelo menos 1 deficiência de todas as capitais e do Distrito Federal, agrupadas nas 5 regiões do Brasil.

Gráfico 1. População com necessidades especiais e sem necessidades especiais. Brasil, 2017.

\section{População PNEs nas capitais Brasileiras}

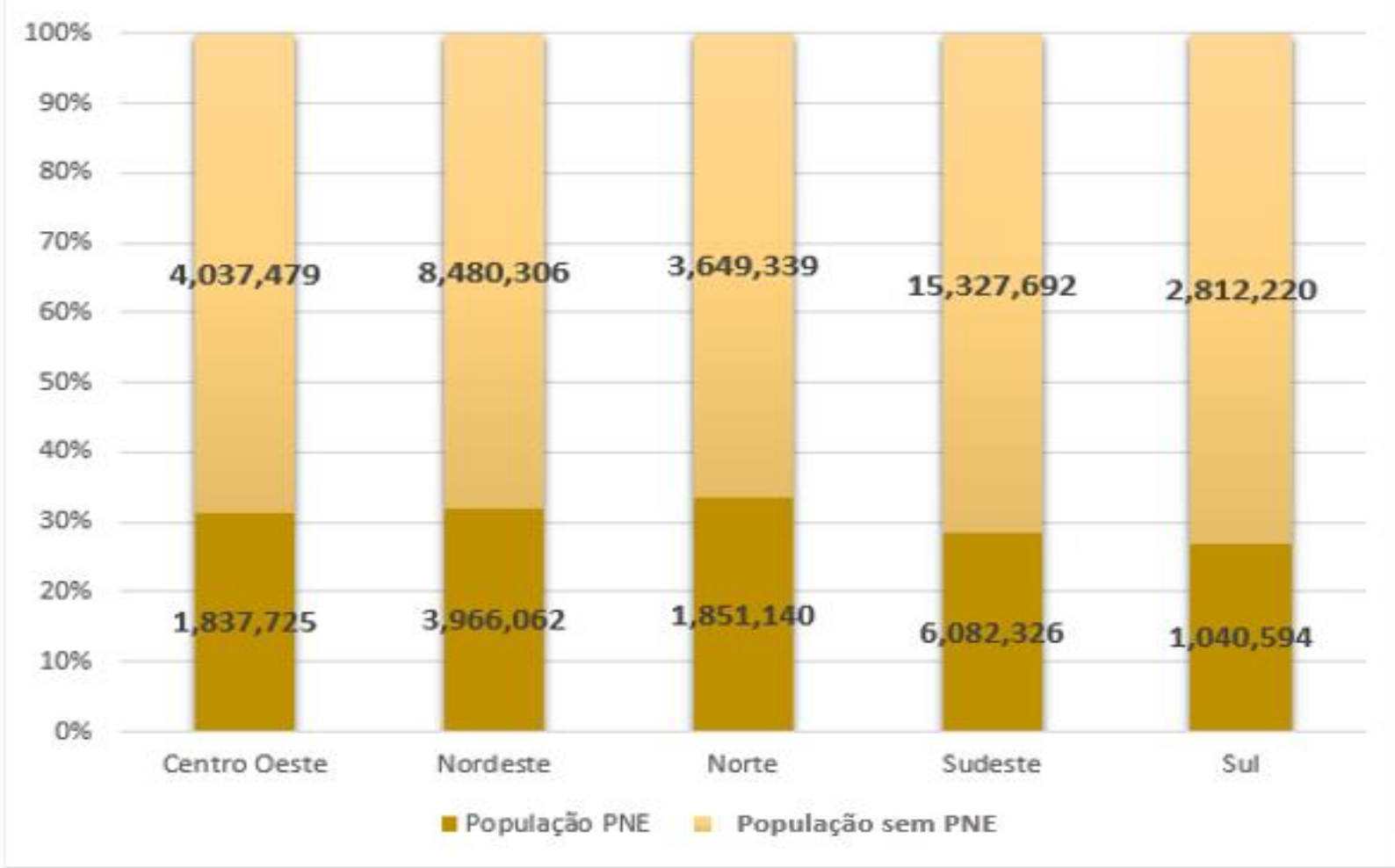

Fonte: Instituto Brasileiro de Geografia e Estatística-IBGE.

De acordo com o Gráfico 1, observa-se que a soma das capitais da região Sul e Sudeste possui a menor proporção de pessoas com pelo menos algum tipo de deficiência em relação à população sem deficiência, abaixo dos $30 \%$ da população total.

Em contrapartida, a soma das capitais de todas as demais regiões está acima dos $30 \%$, destacando-se a região Norte, que tem quase $35 \%$ da sua população com pelos menos uma deficiência em relação à população total.
No Gráfico 2, verifica-se a quantidade de Centros de Especialidades Odontológicas nas capitais brasileiras, agrupados por região. Verifica-se que apenas a região Centro-Oeste possui uma quantidade quase equivalente (pouco mais de 20 unidades) de CEOs que têm a especialidade "pacientes com necessidades especiais" e daqueles que não a têm. Dessa forma, percebe-se que não são habilitados para atendimento dessa parcela da população. Em contrapartida, observa-se uma desproporção dessa relação na região Sul, que 
tem um número muito maior de CEOs não habilitados para atendimento à pessoa com deficiência.

Analisando as capitais, e partindo da relação de que para cada 500 mil habitantes é necessário um CEO habilitado para atendimento às pessoas com deficiência, vê-se que a região Centro-Oeste tem todas as suas capitais e Distrito Federal com número de CEOs PNE suficientes para atendimento da população.

Na região Nordeste também se observa um quadro semelhante. Apenas as capitais do Rio Grande do Norte e Maranhão apresentam o número de CEOs PNE cadastrados no banco de dados do DAB inferior ao necessário diante da sua população de pessoas deficientes.

$\mathrm{Na}$ região Norte, há desproporção entre as capitais, que por vezes têm um número de CEOs PNE muito além da real necessidade, de acordo com a quantidade de pessoas com deficiência e outras, havendo um déficit com a referida habilitação. Destacamse as seguintes capitais: Boa Vista, Manaus e Palmas, que possuem número insuficiente de CEOs habilitados para atendimento de PNE, e as cidades de Porto Velho e Macapá, que tem mais que o dobro de CEOs habilitados para sua demanda.

Avaliando a região onde se encontram as duas maiores metrópoles brasileiras, apenas a cidade de São Paulo, que tem aproximadamente 3.500 .000 pessoas com pelo menos um tipo de deficiência e que, teoricamente, necessitaria de apenas 07 CEOs habilitados, possui 28, o que a torna a capital brasileira com maior número de CEOs desse tipo. Em contrapartida, todas as demais capitais da região Sudeste têm número aquém do necessário para atendimento da sua demanda especializada.

Já a região Sul que tem apenas Florianópolis como destaque positivo, tem um número reduzido de CEOs habilitados. Apesar disto, a quantidade é suficiente para o atendimento de sua população com deficiência.

No Gráfico 3, observa-se as capitais brasileiras e o Distrito Federal agrupados nas suas devidas regiões, quantos CEOs habilitados para atendimento à população com pelo menos uma deficiência há em cada região, e quantos de fato seriam necessários para atingir a proporção de 1 CEO para cada 500 mil habitantes.

Gráfico 2. Número de CEOs Gerais em relação aos CEOs com atendimento à população com Necessidade Especial. Brasil, 2017.

\section{Números de CEOs Gerais x CEOs PNE nas capitais brasileiras}

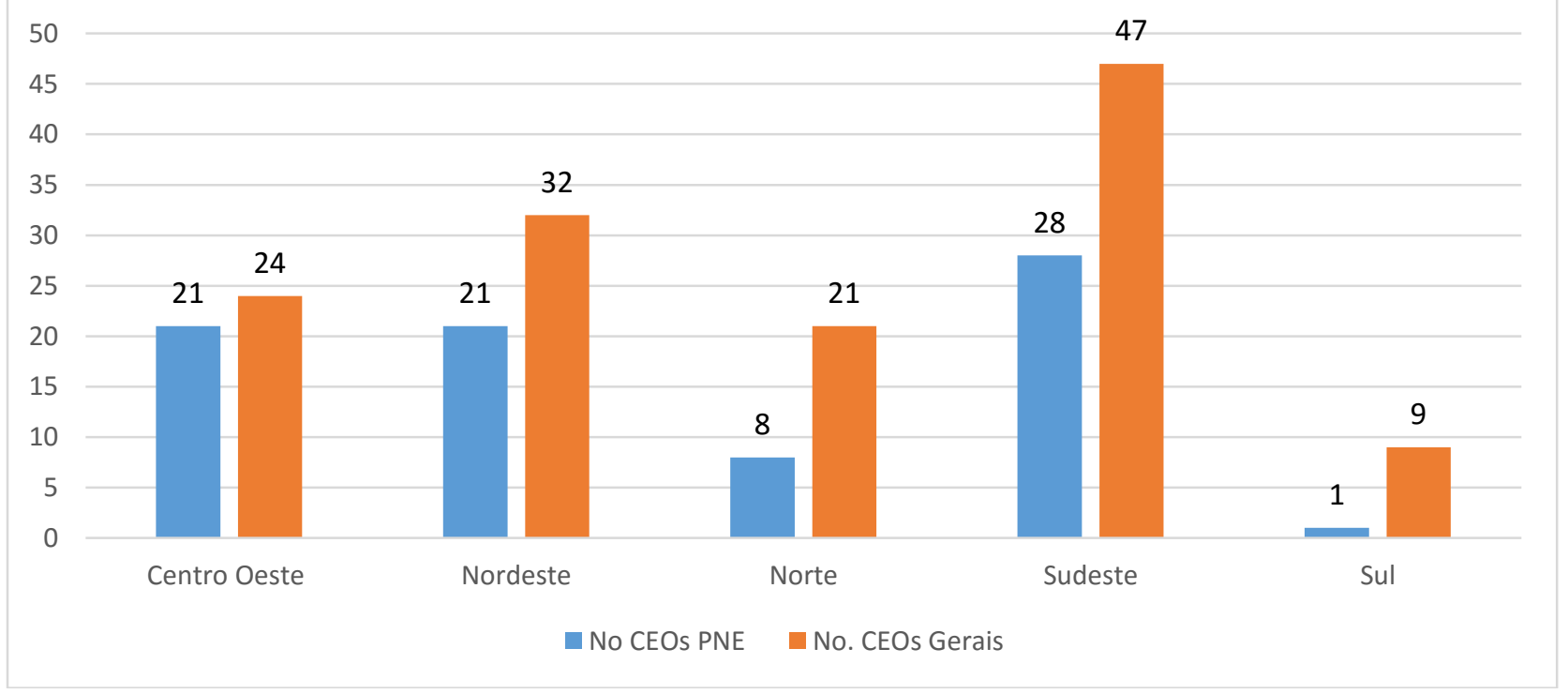


Gráfico 3. Número ideal de CEOs PNE para atendimento nas capitais brasileiras agrupados por região.

\section{Número de CEOs PNE}

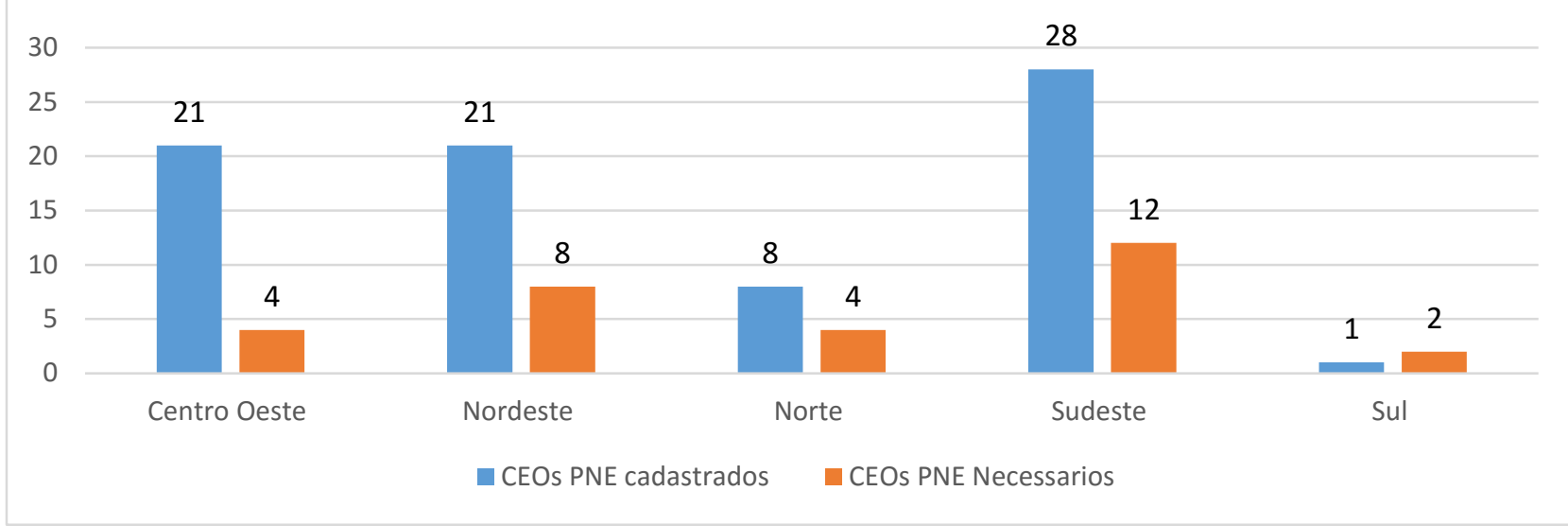

\section{DISCUSSÃo}

De acordo com a Portaria No. 1570, de 29 de julho de 2004, o Ministério da Saúde preconiza que deverá haver um Centro de Especialidades odontológicas para cada grupo de 500 mil habitantes ${ }^{10}$.

Partindo da informação de que cada CEO possui 1 especialista em cada área, se considera uma boa média para cada 500 mil pacientes com algum tipo de deficiência ${ }^{10}$.

É inegável que as Políticas Públicas brasileiras em relação à Odontologia avançaram muito nas ultimas duas décadas $8,9,11$, com a inserção do cirurgiãodentista na Estratégia Saúde da Família, e posteriormente sua inclusão na média complexidade, o que gerou maior suporte e melhor atendimento da Atenção Básica. Mas de acordo com o presente estudo, as capitais brasileiras apresentam uma realidade aquém do esperado em relação à cobertura pelos Centros. Destaca-se, principalmente, as capitais das regiões Norte e Nordeste.

Verifica-se nitidamente o quanto ainda há de se investir e melhorar o atendimento odontológico especializado para a população com deficiência.

Vale salientar também que esse estudo leva em consideração apenas as capitais, deixando de fora grandes centros urbanos que normalmente se encontram no interior dos Estados e municípios menores, que muitas vezes não têm capacidade estrutural, geográfica, e demanda para ter um Centro de
Especialidades Odontológicas. Nesses casos, as capitais apresentam-se como a única solução para referenciar esse tipo de paciente.

Excluindo-se a região Sul, todas as demais possuem CEOs habilitados para o atendimento à pessoa com deficiência em número bem maior que o ideal. No entanto, sofrem de uma má distribuição geográfica entre as capitais, o que demonstra total incompatibilidade da quantidade de Centros como esses em relação ao número de pessoas que precisam de assistência.

Isto faz com que o serviço de atendimento tenha grande folga em algumas cidades e ofereça atendimento precário ou nenhum em outras. Na prática, repercute em elevado tempo de espera para se consultar e/ou remarcar retornos e em sobrecarga do profissional, que algumas vezes não possui especialidade, mas precisa realizar o tratamento ${ }^{12}$.

Nas capitais onde não há CEOs com atendimento especializado para PNEs os usuários são encaminhados para os CEOs que atendem às demais especialidades, sendo os pacientes atendidos em suas necessidades por especialistas Endodontistas, Periodontistas, Cirurgiões e Odontopediatras.

Verifica-se uma deficiência na distribuição dos CEOs que se destinam ao atendimento a Pacientes Portadores de Necessidades Especiais. No entanto, sabe-se que os CEOs devem apresentar um papel de extrema importância social, principalmente 
no que concerne à redução das iniquidades de acesso aos serviços odontológicos especializados, propiciando reduções na morbidade relacionada à saúde bucal ${ }^{13}$. Dessa maneira, enfatiza-se a avaliação em prol de melhorias para o seu funcionamento, e consequentemente, melhoria nos índices da saúde e na qualidade de vida da população.

Estudos anteriores sobre a equidade na distribuição dos CEOs, observaram que a maior parte dos CEOs implantados estava concentrada em municípios de grande porte demográfico e com melhores Índices de Desenvolvimento (IDH) ${ }^{4,14}$, fato observado também neste estudo. Dessa forma, percebese uma relação ainda desproporcional entre o número de pessoas necessitadas e a distribuição dos Centros. Esse fenômeno em que o serviço ofertado não atinge toda a população necessária foi descrito por Hart, que o chamou "Lei da Assistência Reversa"15.

Nas cidades onde não há Centros de Especialidades Odontológicas com PNE, a demanda de atendimento a pacientes com necessidades especiais é diluída na Atenção Básica e nos próprios CEOs não habilitados, quando há condição do paciente ser atendido, ou diretamente na alta complexidade, incluindo os centros hospitalares que possuem atendimento odontológico. Uma pesquisa $^{13}$ mostrou que o desempenho do CEO é influenciado por formas de organizações e pelo gerenciamento dos processos de trabalho e características dos locais onde estão implantados ${ }^{16}$, fato que justifica os resultados obtidos neste estudo.

\section{CONCLUSÃO}

Constatou-se uma grande deficiência no atendimento a pacientes portadores de necessidades especiais na média complexidade. Não necessariamente em relação à quantidade de Centros habilitados que estão distribuídos nas capitais brasileiras e Distrito Federal, mas sim em como esses centros estão distribuídos por entre essas cidades.

Se faz necessário um melhor planejamento por parte das Políticas Públicas, bem como dos Governos Federal e Municipal para uma melhor distribuição desses CEOs de acordo com o número de pacientes que necessitam desse tipo de atendimento especializado.

Isto pois, devido a questões físicas e geográficas, não é possível que eles sejam assistidos na atenção básica ou de média complexidade, caso não haja serviço especializado.

Mais uma vez se vê um grande projeto de política de saúde sendo executado de forma eficaz, mas que precisa de ajustes, planejamento e mais investimentos não apenas nas capitais (que foram o foco do presente estudo), mas também em cidades menores, visando um pronto atendimento a essa parcela da população de forma mais rápida e com qualidade.

Houve um grande salto de qualidade e quantidade de atendimento nos CEOs no início dos anos 2000, que cresceu nos anos seguintes, mas que atualmente não segue o mesmo ritmo de crescimento e alcance para a população que não dispõe de recursos para se locomover até as grandes metrópoles para atendimento especializado.

\section{REFERÊNCIAS}

1. Ministério da Saúde (Br). Secretaria de Atenção à Saúde. Departamento de Atenção Básica. Coordenação Nacional de Saúde Bucal. Diretrizes da Política Nacional De Saúde Bucal. Brasília, DF: Ministério da Saúde; 2004. 2. Ministério da Saúde (Br). Portaria MS/GM no 1.571, de 29 de julho de 2004. Estabelece o financiamento dos Centros de Especialidades Odontológicas - CEO. D.O.U, Brasília, DF, 30 jul; 2004. p. 72, Seção 1.

3. Ministério da Saúde (Br). Portaria no 599, de 23 de Março de 2006. Define a implantação de Especialidades Odontológicas (CEO) e de Laboratórios Regionais de Próteses Dentárias (LRPDs) e estabelecer critérios, normas e requisitos para seu credenciamento [Internet]. Brasília, DF: Ministério da Saúde; 2006 [citado em 07 abril 2017]. Disponível em:

http://bvsms.saude.gov.br/bvs/saudelegis/g m/2006/prt0599_23_03_2006.html.

4. Saliba NA, Nayme JGR, Moimaz SAS, Cecilio LPP, Garbin CAS. Organização da demanda de 
um Centro de Especialidades Odontológicas. Rev Odontol UNESP. 2013; 42(5):317-23.

5. Chaves SCL, Barros SG, Cruz DN, Figueiredo ACL, Moura BLA, Cangussu MCT. Política Nacional de Saúde Bucal: fatores associados à integralidade do cuidado. Rev Saúde Pública. 2010; 44(6):1005-13.

6. Ministério da Saúde (Br), Secretaria de Atenção à Saúde Básica. Programa Nacional de Melhoria do Acesso e da Qualidade da Atenção Básica. Brasília, DF: Ministério da Saúde; 2011.

7. Ministério da Saúde (Br). Portaria GM/MS no 793, de 24 de abril 2012. Institui a Rede de Cuidados à Pessoa com Deficiência no âmbito do Sistema Único de Saúde [Internet]. Brasília, DF: Ministério da Saúde; 2012 [citado em 12 abril 2017]. Disponível em:

http://bvsms.saude.gov.br/bvs/saudelegis/g m/2012/prt0793_24_04_2012.html.

8. Freitas CHSM, Lemos GA, Pessoa TRRF, Araujo MF, Forte FDS. Atenção em saúde bucal: avaliação dos centros de especialidades odontológicas da Paraíba. Saúde Debate. 2016; 40(108):131-43.

9. Rigo L, Basso K, Pauli J, Cericato GO, Paranhos LR, Garbin RR. Satisfaction with life, dental experience and self-perception of oral health among the elderly. Ciênc Saúde Coletiva. 2015; 20(12):3681-8.

10. Ministério da Saúde $(\mathrm{Br})$. Portaria $\mathrm{n}$ o 1570/GM, de 29 de julho de 2004. Estabelece critérios, normas e requisitos para a implantação e habilitação de Centros de Especialidades Odontológicas e Laboratórios Regionais de Próteses Dentárias. D.O.U., Brasilia, jul 2004. Seção 1, p.71-72.
11. Cortellazzi KL. Variáveis associadas ao desempenho de Centros de Especialidades Odontológicas no Brasil. Rev Bras Epidemiol. 2014;17(4):978-88.

12. Frichembruder K, Hugo FN, Hilgert JB. Perfil e percepção de profissionais dos Centros de Especialidades Odontológicas do Rio Grande do Sul. RFO UPF. 2017;22(1):1-6. 13. Machado FCA, Silva JV, Ferreira MAF. Fatores relacionados ao desempenho de Centros de Especialidades Odontológicas. Ciênc Saúde Coletiva. 2015;20(4):1149-63.

14. Goes PSA, Figueiredo N, Neves JC, Silveira FMM, Costa JFR, Pucca Júnior $G$, et al. Avaliação da atenção secundária em saúde bucal: uma investigação nos centros de especialidades do Brasil. Cad Saúde Pública. 2012; 28(Supl):S81-S89

15. Hart JT. The inverse care Law. Lancet 1971; 1(7696):405-12.

16. Herkrath FJ, Herkrath APCQ, Costa LNBS, Gonçalves MJF. Desempenho dos Centros de Especialidades Odontológicas frente ao quadro sociodemográfico dos municípios do Amazonas, Brasil. Saúde Debate. 2009; 37(96):148-58.

CONTRIBUIÇÕES
Renato Carvalho Morais Junior atuou na
concepção, delineamento, coleta de dados e
redação. Laís Guedes Alcoforado de Carvalho
contribuiu no delineamento e redação. Isabella
Lima Arrais Ribeiro participou no
delineamento, análise estatística e redação.
Ricardo Dias de Castro atuou no
delineamento, orientação e supervisão do
artigo.

Como citar este artigo (Vancouver)
Morais Junior RC, Carvalho LGA, Ribeiro ILA, Castro RD. Avaliação nacional dos centros de referência
odontológico da média complexidade. REFACS [Internet]. 2018 [citado em inserir dia, mês e ano de
acesso]; 6(2):166-173. Disponível em: inserir link de acesso. D0I: inserir link do DOI.
Como citar este artigo (ABNT)
MORAIS JUNIOR, R. C. et al. Avaliação nacional dos centros de referência odontológico da média
complexidade. REFACS, Uberaba, MG, v. 6, n, 2, p. 166-173, 2018. Disponível em: <inserir link de
acesso>. Acesso em: inserir dia, mês e ano de acesso. DOI: inserir link do DOI.
Como citar este artigo (APA)
Morais Junior, R. C., Carvalho, L. G. A., Ribeiro, I. L. A. \& Castro, R. D. (2018). Avaliação nacional dos
centros de referência odontológico da média complexidade. REFACS, 6(2), 166-173. Recuperado em:
inserir dia mês e ano de acesso de inserir link de acesso. DOI: inserir link do DOI.

